\title{
Reingeniería en el proceso de gestión e innovación de la asistencia médica hospitalaria
}

Del Castillo Rueda A, Khosravi Shahi P. Reingeniería en el proceso de gestión e innovación de la asistencia médica hospitalaria. An Med Interna (Madrid) 2005; 22: 509-510.

El incremento del gasto farmacéutico y hospitalario, el aumento de la demanda de una población envejecida y la generosidad del sistema amenazan el futuro de la sanidad. Las organizaciones sanitarias operan en entornos específicos y generan servicios específicos pero son empresas (1) y por tanto pueden y deben ser gestionados con los mismos principios y criterios de dirección. Una cultura empresarial ganadora debe aspirar a gobernar el caos, haciendo de la anticipación y la flexibilidad las señas de identidad que permitan a la organización comprometerse en el desafío de la innovación permanente.

El nuevo y cambiante entorno demográfico, sociológico, cultural y tecnológico debe interpretarse como oportunidades de mejora para la asistencia médica hospitalaria y el papel de los médicos como agentes en busca de un cambio radical centrado en "qué queremos ser" y no en "qué somos". La reingeniería en las instituciones de salud es un proceso de cambio creativo, de reinventar y renovar las estructuras, los procesos, los sistemas y las maneras de medir los resultados y el desempeño de la organización. La reingeniería está enfocada a los resultados, que se miden de acuerdo con la satisfacción del usuario (2,3).

La gestión clínica pretende optimizar los resultados de la actividad clínica, permitir que los profesionales tengan capacidad para organizarse y diseñar estrategias para la mejora de los resultados y la búsqueda de la satisfacción del paciente. Los modelos de gestión clínica buscan objetivos de descentralización de la responsabilidad, mejora de las relaciones entre profesionales, usuarios y directivos y control de costes, vinculando la financiación a la actividad asistencial. La esencia de la gestión clínica es reducir la brecha entre eficacia -lo que puede conseguirse- y eficiencia -los resultados-, mientras que la mejora de la calidad pasa por cruzar el abismo entre el mejor cuidado posible y el cuidado rutinario diario (4).

Aunque no habrá buena gestión sanitaria sin buena medicina, tenemos que admitir que tampoco puede existir buena medicina sin buena gestión sanitaria (5). Formados en la idea de que hay que hacer buena medicina basada en nuestra formación y conocimientos así como en principios éticos, es preciso añadir a nuestra práctica clínica elementos de gestión, calidad y seguridad asistencial. De tal manera que nuestro compromiso profesional y social sea ofertar a nuestros pacientes los mejores resul- tados posibles (efectividad) con medidas útiles (eficacia) y con los menores inconvenientes y costes (eficiencia) (4).

La clínica y la gestión deben buscar un punto de encuentro para compartir conocimientos y proporcionar la mejor medicina posible con los recursos disponibles teniendo en cuenta que la función de los gestores es evaluar intervenciones y proporcionar al médico información completa de la máxima calidad y la de los clínicos es evaluar pacientes y utilizar la información de la forma más idónea (6).

La visión clásica de un servicio limitado por un espacio físico, manejado desde la perspectiva de una especialidad con actividad y carga asistencial correlacionada con el número de camas y éste con los recursos humanos, debe dejar paso a una unidad funcional sin límites físicos, centrada en resolver los problemas del paciente, gestionada por una organización horizontal y participativa con equipos multidisciplinarios y que utilice con criterios de calidad los recursos disponibles.

La atención debe pivotar alrededor del paciente (servicio sanitario) y no alrededor del espacio donde se presta este servicio (cama hospitalaria). Además el alta hospitalaria no debe ser sinónimo de cierre de episodio ya que éste puede realizarse en otras áreas. Así frente a la hospitalización tradicional o convencional, y la disminución de camas de agudos, se han generado alternativas que permiten optimizar el empleo de cama hospitalaria y potenciar su uso adecuado: unidades de hospitalización domiciliaria, hospitales de día, unidades de diagnóstico, unidades de corta estancia (salas de alta rápida) y unidades de atención por procesos asistenciales (7).

La conducta médica basada en el modelo de ética médica, supone que el médico está fundamentalmente motivado por su deseo de hacer todo lo que sea posible en bien del paciente, al margen del coste que ello suponga. El reto al que se enfrentan los sistemas y organizaciones sanitarias es cómo desarrollar los incentivos y motivaciones apropiados para que en la conducta basada en la ética profesional se introduzca la dimensión del coste como un factor relevante en la toma de decisiones (8). La medicina gestionada permite alcanzar el objetivo ético de la excelencia (9) convirtiendo al clínico responsable en un gestor sanitario ensamblando los fundamentos éticos de la medicina (principio éticos de beneficencia, no-maleficen- 
cia, autonomía y justicia) con el logro de los mejores cuidados sanitarios posibles para los enfermos $(10,11)$.

Los programas de gestión tienen muchos puntos en común con los de calidad ya que ambos persiguen en definitiva lo mismo: hacer bien el trabajo (12). La gestión de calidad total supone la búsqueda de la excelencia, ligada a la competitividad, en todo lo referente a resultados y rendimientos de una organización mediante la autoevaluación, resaltando puntos fuertes y áreas de mejora y contrastándolo con un modelo de excelencia: el modelo EFQM (European Foundation for Quality Management) que se usa como base para el diagnóstico del sistema de gestión y evaluación del progreso hacia la excelencia (13).

En este modelo de calidad el primer criterio es el liderazgo con la definición de cómo los lideres desarrollan y facilitan la consecución de la misión y la visión, desarrollan los valores necesarios para alcanzar el éxito e implantan todo ello en la organización mediante las acciones y los comportamientos adecuados, estando implicados personalmente en asegurar que el sistema de gestión de la organización se desarrolla e implanta (14).

Por fín, la asistencia medica hospitalaria debe ser además segura para lo que es preciso realizar prevención de accidentes asistenciales así como análisis de accidentes y errores. La primera alerta de seguridad se enunció en 2002 y es la inadecuada identificación de pacientes, con estrategias de mejora con la utilización del brazalete identificativo y de un sistema de código de barras (15). Actualmente se han publicado otras cuatro alertas más sobre introducción de prácticas seguras ya establecidas que se priorizan en función de la evidencia científica de mayor impacto y coste de aplicación: infravaloración de traumatismo, riesgo de potasio intravenoso, cirugía en lugar erróneo e inmovilización de pacientes, y que suponen un nuevo reto para que nuestra actividad clínica sea una práctica asistencial segura (16).

La innovación competitiva significa transformación que implica ruptura y se lleva mal con la cultura cortoplacista de no asumir retos y pensar que el paso del tiempo lo arreglará todo. Conlleva una cultura de asunción de riesgos que tiene su principal enemigo en la burocracia. Esta innovación competitiva que descansa sobre valores surge de la confluencia de tres elementos básicos: tecnología (tecnologías de la información y las comunicaciones: TIC), conocimiento (información, formación y comunicación) y cooperación (17).

La reingeniería en las instituciones de salud supone el empleo de métodos de trabajo con metodología específica y participativa para identificar problemas, proponer intervenciones de mejora y seleccionar resultados, permaneciendo abiertos para incorporar mejoras. Los grupos participativos liderados por profesionales con experiencia clínica y de gestión actuaran junto a estamentos sanitarios, asociaciones de vecinos, enfermos y familiares $(2,18)$.

La actividad clínico-asistencial es un conjunto de relaciones que se rigen por principios de tipo cliente-proveedor (médicopaciente y médico-organización) (19) por lo que la capacidad de transformar la asistencia debe ser de una manera sostenible y no a cualquier precio, con diálogo entre las partes interesadas y con preocupación además por la satisfacción laboral de los empleados, sus posibilidades de desarrollo profesional y el sistema de recompensas. El fin será conseguir una atención médica hospitalaria excelente aplicando medidas sobre la base de criterios éticos, de gestión, calidad y seguridad, con la participación en las decisiones de todos los estamentos implicados, mejorando las relaciones, realizando un seguimiento del proceso y comparando los resultados con los de organizaciones externas para mejorar el rendimiento global mediante actividades de "mejores prácticas" (benchmarking) (3).

\section{A. DEL CASTILLO RUEDA, P. KHOSRAVI SHAHI}

\section{Servicios de Medicina Interna II y Oncología Médica*. Hospital General Universitario Gregorio Marañón. Madrid}

\section{Bibliografía}

1. Asenjo MA. El hospital como empresa. Med Clin (Barc) 1991; 96: 780-783.

2. Cerrada Bravo T. Reingeniería en sistemas de salud. Rev Med IMSS 2002; 40: 127-135

3. Bermudo L, Navarro A, Baena V, Fuentes JL, Juan F. Reingeniería de empresa en el hospital. Todo Hospital 2002; 183: 24-32

4. Del Castillo Rueda A, De Portugal Álvarez J. Proyecto técnico de gestión y funcionamiento de la unidad asistencial de Medicina Interna. An Med Interna (Madrid) 2004; 21: 31-38.

5. Ortún Rubio V. Gestión clínica y sanitaria. Masson S.A. Barcelona 2003.

6. Sacristán JA, Rovira J, Ortún V, García-Alonso F, Prieto L, Antoñanzas F. Utilización de las evaluaciones económicas de intervenciones sanitarias. Med Clin (Barc) 2004; 122: 789-795.

7. Torres Salinas M, Capdevilla Morel JA, Arnario García P, Montull Morer S y grupo de trabajo de los Servicios de Medicina Interna de los Hospitales de Cataluña. Alternativas a la hospitalización convencional en medicina interna. Med Clin (Barc) 2005; 124: 620-626.

8. Ruiz Iglesias L. Claves para la gestión clínica. Mc Graw-Hill/Interamericana de España, S.A.U. Madrid 2004.

9. Jiménez J. Manual de gestión para jefes de servicios clínicos. You\&Us, S.A. Madrid 1997.

10. Llano J del, Millán J. Ética y gestión sanitaria. Med Clin (Barc) 2002; 118: 337-338.
11. Quintana O. Los objetivos de la medicina. Rev Calidad Asistencial 2003; 18: 132-135.

12. Millán J. La gestión sanitaria y clínica: ¿una necesidad? ¿una moda?. Hospital General 2003; 3: 9-11.

13. Mirá JJ, Rodríguez J, Peset R, Buil JA, Vitaller J, García A et al. Buenas prácticas para el hospital del siglo XXI. Todo Hospital 2002; 192: 781787

14. Oteo LA, Silva D, Garrido J. Desarrollo de los criterios del modelo europeo de gestión de calidad en hospitales públicos. Gestión Hospitalaria 2000; 11: 127-143

15. Alertas de seguridad. Centro de Investigación para la Seguridad Clínica de los Pacientes. Fundaciones Avedis Donabedian y Mapfre Medicina. [www.fadq.org]

16. Aibar-Remón C. La seguridad clínica: pequeños pasos y grandes palabras. Rev Calidad Asistencial 2005; 20: 183-184.

17. Larrea JL. Empresas: El desafío de la innovación. El País Negocios. Domingo 25 de septiembre de 2005, p. 15.

18. Vázquez G, Benito S, Ruiz D, Domingo P, Domingo G, Soler D et al. La propuesta de rediseño del Servicio de Medicina Interna del Hospital de la Santa Cruz y San Pablo. Rev Calidad Asistencial 2004;19:20-26.

19. Cosialls D, Gil MA. Gestión clínica. Una nueva óptica: cliente-proveedor. Todo Hospital 2002; 192: 761-768. 